\title{
BMJ Open Fluids in Sepsis and Septic Shock (FISSH): protocol for a pilot randomised controlled trial
}

\author{
Bram Rochwerg, ${ }^{1,2}$ Tina Millen, ${ }^{1}$ Peggy Austin, ${ }^{2}$ Michelle Zeller, ${ }^{1}$ \\ Frédérick D'Aragon, ${ }^{3}$ Roman Jaeschke, ${ }^{1,2}$ Marie-Hélène Masse, Sangeeta Mehta, ${ }^{4}$ \\ Francois Lamontagne, ${ }^{3}$ Maureen Meade, ${ }^{1,2}$ Gordon Guyatt, ${ }^{1,2}$ Deborah J Cook, ${ }^{1,2}$ \\ on behalf of the Canadian Critical Care Trials Group
}

To cite: Rochwerg B, Millen T, Austin $\mathrm{P}$, et al. Fluids in Sepsis and Septic Shock (FISSH): protocol for a pilot randomised controlled trial. BMJ Open 2017;7:e017602. doi:10.1136/ bmjopen-2017-017602

- Prepublication history and additional material is available. To view please visit the journal (http://dx.doi.org/ 10.1136/ bmjopen-2017-017602)

Received 3 May 2017 Revised 15 June 2017 Accepted 26 June 2017

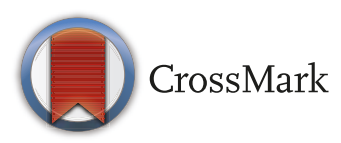

${ }^{1}$ Department of Medicine, Division of Critical Care, McMaster University, Hamilton, Ontario, Canada

${ }^{2}$ Department of Health Research Methods, Evidence and Impact, McMaster University, Hamilton, Ontario, Canada

${ }^{3}$ Centre de Recherche du Centre Hospitalier Universitaire de Sherbrooke et Faculté de

Médecine et des Sciences dela

Santé, Université de Sherbrooke,

Sherbrooke, Quebec, Canada

${ }^{4}$ Mount Sinai Hospital and Interdepartmental Division of Critical Care Medicine, University of Toronto, Toronto, Ontario, Canada

Correspondence to

Dr Bram Rochwerg; rochwerg@ mcmaster.ca

\section{ABSTRACT}

Introduction Observational evidence suggests

physiological benefits and lower mortality with lower chloride solutions; however, $0.9 \%$ saline remains the most widely used fluid worldwide. Given uncertainty regarding the association of lower chloride on mortality, it is unlikely that practice will change without direct randomised clinical trial (RCT) evidence. This pilot RCT will investigate the feasibility of a large-scale trial directly comparing low chloride with high chloride fluids in patients with septic shock.

Methods and analysis This is a randomised, concealed, blinded parallel-group multicentre pilot trial. We will include adult critically ill patients with septic shock, defined as ongoing hypotension despite $1 \mathrm{~L}$ of fluid, or a serum lactate $>4 \mathrm{mmol} / \mathrm{L}$, who are within 6 hours of hospital presentation or rapid response team activation. We will exclude patients if they have an aetiology of shock other than sepsis, if they have acute burn injury, elevated intracranial pressure, intent to withdraw life support or previous enrolment in this or a competing trial. Following informed consent, patients will be randomised to a low chloride fluid strategy or a high chloride fluid strategy for the duration of their ICU stay or until 30 days postrandomisation. Clinicians, patients, families and research staff will be blinded. The primary outcome for this trial will be feasibility, assessed by consent rate, recruitment success and protocol adherence. Patientimportant clinical outcomes include mortality, receipt of renal replacement therapy, intensive care unit and hospital lengths of stay and surrogate outcomes of incidence of acidosis, hyperkalaemia and acute kidney injury.

Ethics and dissemination This pilot trial will test the feasibility of conducting the main trial, which will examine the effect of high versus low chloride fluids in patients with septic shock on patient-important outcomes.

Trial registration number NCT02748382, registered 8 April 2016.

Protocol date 1 July 2016.

\section{BACKGROUND}

In addition to antimicrobials, replacement of intravascular fluid is the mainstay of treatment for patients with septic shock to maintain organ perfusion and oxygen delivery to the

\section{Strengths and limitations of this study}

- Pragmatic design as clinicians may prescribe either crystalloids or albumin fluid types as they would normally as part of practice, patients will receive fluid with chloride content corresponding to allocated study arm.

- Focus specifically on patients with septic shock and those at high risk for large volume fluid resuscitation.

- Given the goals of feasibility, we will not be able to make conclusions related to efficacy from this pilot randomised controlled trial.

periphery. ${ }^{1}$ Several fluid solutions, categorised as either crystalloids or colloids, are available for resuscitation. Each fluid has unique properties, including tonicity, $\mathrm{pH}$ and osmolality, depending on its specific electrolyte and molecular composition. ${ }^{2}$ The most commonly used isotonic crystalloid worldwide is $0.9 \%$ normal saline (saline) which contains a supraphysiological concentration of chloride. Some other isotonic crystalloid solutions contain an organic anion (eg, lactate, maleate, acetate) and therefore have a lower chloride content more closely resembling that of human plasma (eg, Ringer's Lactate and Ringer's Acetate).

Colloids, in contrast, contain large-molecular weight compounds that remain in the intravascular space for a longer period of time. A potentially crucial factor rarely considered is the chloride content of the crystalloid in which a specific colloid is dissolved. ${ }^{3}$ The clinical effects of hyperchloraemia resulting from intravenous fluid replacement are unclear. A before-and-after single-centre observational cohort study showed a decrease in renal failure in patients receiving low chloride fluids compared with high chloride. ${ }^{4}$ Results from a network meta-analysis (NMA) of randomised controlled trials 
(RCTs) including indirect evidence suggested balanced crystalloids may result in lower mortality (NMA OR 0.78; 95\% credible interval (CrI) 0.58 to 1.05 ) and a decreased receipt of renal replacement therapy (NMA OR 0.85; $95 \%$ CrI 0.56 to 1.30 ) relative to saline; however, the evidence warranted only low certainty. ${ }^{5}$ A cluster randomised crossover pilot trial from four intensive care units (ICUs) in New Zealand found no difference in acute kidney injury (AKI) when comparing low chloride (Plasma-Lyte) with high chloride crystalloid $(0.9 \%$ saline $)$; however, patients had low APACHE II scores (mean 14.1), received only a small amount of study fluid (median $2 \mathrm{~L}$ throughout the trial period) and this pilot trial was underpowered to detect modest treatment effects. ${ }^{6}$

Given the residual uncertainty regarding the effect of using high versus low chloride fluids in sepsis, and that saline remains the most widely used fluid worldwide, a large RCT is necessary to investigate the effect of using different fluids patient important outcomes such as mortality and AKI. Herein, we report the protocol for the Fluids in Septic Shock (FISSH) Pilot Trial, the objective of which is to evaluate the feasibility of performing a larger RCT examining this question.

\section{METHODS \\ Design}

This is a randomised, concealed, blinded parallel-group multicentre pilot trial. The primary outcome is feasibility, assessed by three outcomes: consent rate $(>70 \%)$, recruitment success ( 50 patients total at the three centres and greater than one patient enrolled/site/month) and protocol adherence (receipt of study fluid for $>75 \%$ of all intravenous fluids administered in the ICU excluding blood products and medication infusions). We plan to enrol patients at three Canadian University-affiliated academic teaching hospitals. The Juravinski Hospital associated with McMaster University in Hamilton, Ontario, Centre Hospitalier Universitaire de Sherbrooke associated with Sherbrooke University in Sherbrooke, Quebec and Sinai Health System associated with the University of Toronto. The study will be conducted through collaboration with the Canadian Critical Care Trials Group (CCCTG), a network of investigators and research coordinators who conduct investigator-initiated research.

\section{Population}

We will include patients who are: (1) $\geq 16$ years of age; (2) require fluid resuscitation for refractory hypotension (systolic blood pressure $<90 \mathrm{~mm} \mathrm{Hg}$ or mean arterial blood pressure $<65 \mathrm{~mm} \mathrm{Hg}$ after $1 \mathrm{~L}$ bolus over $\leq 1$ hour) or organ hypoperfusion (serum lactate $>4 \mathrm{mmol} / \mathrm{L}$ ); (3) suspected to have infection as the aetiology of hypotension; (4) within 6 hours of hospital admission or critical care response team consultation and (5) admitted to ICU.

Patients will be excluded if they have intracranial bleed or intracranial hypertension during this hospital admission, acute burn injury ( $>10 \%$ of body surface area), bleeding/haemorrhage as likely cause of hypotension, a plan in place to change goals of care to palliation, were previously enrolled in FISSH or a competing trial, have been transferred from another hospital or facility, were admitted directly from the operating room or postanaesthetic care unit.

\section{Eligible non-randomised patients}

We will record all patients who were eligible but not randomised for any of the following reasons: (1) patient or substitute decision-makers (SDM) declined consent; (2) patient is unable to consent and SDM is not available; (3) ICU physician declined enrolment and (4) any other reasons.

\section{Informed consent}

We have obtained research ethics board (REB) approval at each of the participating centres. Given the need to enrol patients in an expedited manner, the fact that most patients will not be capable of providing consent at the time of study entry and that both the experimental and control study fluids are currently considered the standard of care, we will use a deferred consent model. Patients will be enrolled in the study and consent will subsequently be obtained from the SDM or the patient, or both, in a timely manner (ideally within 72 hours). Precedent for a deferred consent model exists in a study examining the use of emergency resuscitative fluids in Canadian centres. ${ }^{7}$

\section{Randomisation and allocation concealment}

Once the research coordinator has identified an eligible patient, she/he will use the web-based system www. RANDOMIZE.NET to randomise patients. Patients will undergo concealed randomisation, stratified by centre, in a 1:1 schedule to either the lower chloride fluids group or the higher chloride fluids group. The web-based system will generate email alerts to the local research pharmacist and the blood bank with information regarding patient allocation. The pharmacist and transfusion specialist will then ensure the study patients receive their assigned study fluid. Research coordinators and the bedside clinical team will not be aware of allocation.

\section{Experimental procedures}

Enrolled patients will be allocated to either a high or low chloride fluid strategy. Within the allocation, clinicians may order crystalloid or albumin as they normally would in routine clinical practice, the decision regarding which to administer will be left to the clinician's discretion. Only once ordered by the clinical team, study patients will receive fluid (either crystalloid or albumin) with a chloride content corresponding to their allocated arm. The allocated fluid will be administered immediately after randomisation, continued until discharge from the ICU, death or until 30 days after enrolment, whichever comes first. Patients will receive the allocated fluid type for both resuscitation and maintenance infusions. Blood products and fluids used for medication infusions will be recorded but will not be protocolised. 
Given that most clinicians use crystalloids as the initial resuscitation fluid in patients with septic shock, $1 \mathrm{~L}$ bags of study crystalloid will be readily available on carts in relevant patient care areas. Once a patient is randomised, the cart containing crystalloids consistent with their allocated group will be left close to the patient's room to facilitate administration by the bedside nurse. Saline of $0.9 \%$ will be used as crystalloid for those randomised to high chloride fluid (chloride concentration $154 \mathrm{mmol} / \mathrm{L}$ ) while Ringer's Lactate will be used as crystalloid for those in the low chloride arm (chloride concentration $110 \mathrm{mmol} / \mathrm{L}$ ).

Plasbumin 5\% (Grifols, chloride concentration $110 \mathrm{mmol} / \mathrm{L}$ ) will be used as the low chloride albumin and Octalbin 5\% (Octapharma, chloride concentration $137 \mathrm{mmol} / \mathrm{L}$ ) will be used as the high-chloride albumin. The blood bank at each centre will receive a list of enrolled patients and their allocation, and this list will be updated each time a new patient is enrolled. The appropriate albumin solution, blinded in appearance to chloride content, will be supplied to the patient care area for infusion only when prescribed by the bedside physician. All albumin products have Health Canada approval for patient use and are available at all Canadian hospitals through order from Canada Blood Services.

Open-label crystalloid or albumin products will not be permitted in the ICU except as explicitly ordered by the treating physicians. Research coordinators will document all open-label fluid use, their indications and reasons for protocol non-adherence. All other aspects of patient care will be left to the discretion of the treating physicians. After 30 days in ICU or on discharge from the ICU, physicians will use open-label fluid at their discretion.

\section{Blinding}

Patients, families and all bedside clinicians (nurses, allied health providers and physicians) will be blinded to study allocation. All study fluids, whether containing a lower or higher chloride concentration will be identical in appearance, consistency and packaging except for the identifying serial number found on the individual label. Bags of saline and Ringer's Lactate look identical and the product label will be covered with opaque study labels or opaque tape (see online supplementary material). The $5 \%$ albumin bottles also appear identical between the two manufacturers and will be covered by opaque bags and tape to ensure the blinding of bedside staff. Local pharmacy and blood bank staff will ensure that patients receive their allocated fluid and thus will not be blinded. We will also ensure blinding of research staff, site investigators, outcome adjudicators and data analysts.

\section{Outcomes}

Consent rate-we will consider the consent rate adequate if more than $70 \%$ of SDMs or patients when approached for consent choose to participate. This will be calculated as the proportion of SDMs or patients approached who consent. Given our plan for deferred consent, there will be some lag time between study enrolment and consent.
Research coordinators will be supplied with consent scripts and employ consent tools developed by our group. ${ }^{8}$ If a patient or SDM chooses to withdraw consent, the data collected up until that point will be retained for the analysis under the original REB-approved deferred consent model. Reasons for declining to participate will be recorded. The study steering committee will regularly review the consent rate, and, if necessary, barriers will be discussed and interventions undertaken to improve the consent rate.

Recruitment-successful recruitment will be defined as achieving enrolment of 50 patients total and greater than one patient enrolled/site/month. All excluded patients, eligible non-randomised patients (including physician refusals and reasons) will be recorded. Screening logs will be reviewed from the three participating centres by the steering committee on a monthly basis to determine whether any modifications to the protocol might be warranted or to address implementation challenges if needed. Barriers to enrolment will be discussed, and if necessary, strategies to improve enrolment will be operationalised.

Protocol adherence-successful adherence will be defined as patients receiving at least $75 \%$ study fluid of all intravenous fluid administered in the ICU excluding blood products and medication infusions. This threshold was chosen based on discussion within the steering committee and after examining other pilot studies of complex interventions. ${ }^{9}{ }^{10}$ Strategies will be employed a priori to facilitate compliance. As described above, crystalloid study carts will be prepared, stocked regularly and left in a convenient location for nurses and physicians, selected with input from bedside staff. Fluids will be labelled with complex serial numbers corresponding to each patient's allocated study arm to help avoid unblinding. Research coordinators will document all fluids that patients receive including protocol violations. A taxonomy of reasons for non-adherence will be developed to distinguish deviations for clinical reasons from true protocol violations. The steering committee will conduct regular review of protocol adherence. Protocol violation reports will be sent to each centre throughout the feasibility trial to provide real-time feedback; further behavioural strategies will be employed to improve adherence if needed.

Clinical outcomes-Clinical outcomes in the FISSH pilot trial will reflect outcomes for the main trial which will include: hospital mortality, RRT use for AKI during 30-day period postrandomisation (including continuous renal replacement therapy or conventional haemodialysis), proportion of patients with RIFLE stage renal failure, ${ }^{11}$ 30-day mortality, ICU and hospital length of stay (censored at 30 days), ventilator-free days in the first 30 days postrandomisation and safety outcomes (acidosis and electrolyte imbalance postrandomisation). 


\section{Sample size calculation}

Sample size for the pilot was calculated using a 95\% CI approach examining protocol adherence. The feasibility threshold $(75 \%)$ and an expected adherence rate $(95 \%)$ were determined a priori. Using a power of $80 \%$, if the observed adherence rate is $95 \%$ we will be able to exclude $75 \%$ adherence or lower using a sample size of at least 47 patients. To be conservative, we will plan for 50 patients (approximately 25 per study arm). This number of patients will allow us to assess the three feasibility objectives including recruitment parameters according to our a priori feasibility outcomes in a cost-effective manner.

\section{Data collection}

Trained research staff at each centre will collect the data. Staff will complete paper case report forms (CRFs) which they will transcribe into web-based e-CRFs (REDCap - http://www.project-redcap.org) that are encrypted and password protected. All CRFs will be pretested and edited for clarity and ease of use prior to the trial initiation (see online supplementary material). Baseline data will include eligibility criteria, baseline demographics, admitting diagnosis, APACHE II admission score and multiple organ dysfunction score (MODS). While patients remain in the ICU, daily data collection will include MODS, haemodynamics, ventilator requirements, all fluid administered (including study, non-study and blood products), use of renal replacement therapy and other relevant bloodwork. Cointerventions will also be captured including administration of vasopressors/ inotropes, corticosteroids, bicarbonate and diuretics. We will also document length of stay in the ICU and hospital, ICU readmission and mortality.

The web-based eCRFs will allow for data validation, realtime consistency checks and frequent audits to ensure entered data are complete and accurate. The paper CRFs will be retained at each centre for any queries. The Methods centre will be responsible for managing the database and quality assurance using anomaly searches and logic checks. Timely data entry at each centre will be encouraged by the Methods centre to minimise missing data and ensure timely resolution of queries. CRFs will be kept for the duration as required by local regulatory bodies.

\section{Data analysis}

Analysis of the three feasibility outcomes does not require analysis by group. Clinical outcomes will not be analysed by study arm to avoid overinterpretation; instead, clinical outcomes will be presented as means or proportions in both arms combined. No interim analysis or subgroup analyses are planned for this pilot trial.

We intend to run this as an internal pilot and roll these patients into the main FISSH trial pending funding and meeting feasibility targets. For the main FISSH trial, a blinded biostatistician will perform all analyses based on the intention-to-treat principle. The baseline characteristics comparing balanced fluid and unbalanced fluid groups will be reported using means (and SD), medians (and interquartile ranges) or proportions as indicated. Dichotomous outcomes will be reported using OR and $95 \%$ CIs and calculated using logistic regression. Non-parametric testing, the Mantel-Cox log rank test, will be used for the continuous outcomes of ICU length of stay, hospital length of stay and ventilator-free days given when the data are not normally distributed. These continuous variables will be censored at 90 days. An independent t-test will be used to compare the means of the safety outcomes (serum $\mathrm{K}$, serum $\mathrm{pH}$ ) between the two groups and mean difference with $95 \%$ CIs and $p$ values will be reported. A $p$ value $<0.05$ will be considered statistically significant for all outcomes.

\section{DISCUSSION}

Current evidence suggests that albumin and crystalloid, but not starches, represent reasonable choices for fluid resuscitation in sepsis. ${ }^{1}$ Previous studies evaluating resuscitative fluids have limited the intervention to single fluid types in each experimental arm, which is a study design that is incongruent with real-life practice. The FISSH protocol reflects a design that is novel yet practical, overcoming the applicability limitation of prior trials by allowing physicians to prescribe either colloid or crystalloid in the ICU, as they perceive is clinically indicated. The chloride content of both types of fluid will, however, correspond to their randomly allocated group, either higher or lower chloride. This unique design will allow physicians the flexibility to prescribe albumin or crystalloid, decrease the chance of contamination with non-trial fluid, maximise the applicability of the results, maximise the extent to which results will influence clinical practice and increase the likelihood of successful trial completion.

Before embarking on a larger RCT comparing low-chloride with high-chloride fluids for patients with septic shock that is adequately powered to evaluate patient-important outcomes, a pilot trial is necessary. Our biggest perceived threat to the feasibility of this pilot trial is protocol adherence. We have taken as many a priori steps as possible to enhance protocol adherence including preprinted study order sets, educational sessions with bedside staff and convenient bedside fluid carts to facilitate study fluid administration. Results of this pilot trial will demonstrate whether it is possible to deliver the trial interventions as outlined. Achieving our threshold consent rate will demonstrate that the trial is acceptable to patients, families and clinicians. Finally, recruitment parameters will help to estimate the number of centres, period of time and resources that may be needed for efficient conduct of the main trial.

Despite a large body of literature, practice regarding fluid administration in septic shock remains varied and controversial. ${ }^{12-16}$ Study investigators in consultation with the Canadian Critical Care Trials Group will 
consider the results of the FISSH pilot trial, along with any other emerging external evidence (NCT02721654, NCT02875873), to plan the sample size of the trial powered to detect effects on clinically important outcomes. Despite these similar planned studies, we believe our trial is unique given our focus on sepsis only, the inclusion of albumin into the allocated chloride strategy and the enrolment of a sick cohort at high risk for mortality and multiorgan failure. The larger FISSH trial has the potential to inform the management of patients with sepsis and septic shock worldwide.

Acknowledgements This pilot trial is supported by the Canadian Critical Care Trials Group (CCCTG), and we would like to acknowledge Dr Sean Bagshaw for his internal peer review of this manuscript on behalf of the CCCTG. Dr. Rochwerg is supported by an early career research award (AFP, Department of Medicine, McMaster University).

Contributors BR conceived the idea and drafted the protocol. TM, PA, MZ, FD'A, RJ, M-HM, SM, MM, GG, DJC all provided input and revision on the protocol which were then incorporated. BR drafted the manuscript and TM, PA, MZ, FD'A, RJ, M-HM, SM, MM, GG, DJC reviewed and revised. All authors approved the final version for submission.

Funding The FISSH pilot is funded by the Hamilton Health Sciences-New Investigator Fund.

Competing interests None declared.

Patient consent Obtained.

Ethics approval Hamilton Integrated Research Ethics Board.

Provenance and peer review Not commissioned; externally peer reviewed.

Open Access This is an Open Access article distributed in accordance with the Creative Commons Attribution Non Commercial (CC BY-NC 4.0) license, which permits others to distribute, remix, adapt, build upon this work non-commercially, and license their derivative works on different terms, provided the original work is properly cited and the use is non-commercial. See: http://creativecommons.org/ licenses/by-nc/4.0/

C Article author(s) (or their employer(s) unless otherwise stated in the text of the article) 2017. All rights reserved. No commercial use is permitted unless otherwise expressly granted.

\section{REFERENCES}

1. Rhodes A, Evans LE, Alhazzani W, et al. Surviving sepsis campaign: International Guidelines for Management of Sepsis and Septic Shock: 2016. Intensive Care Med 2017;43:304-77.

2. Rochwerg B, Włudarczyk A, Szczeklik W, et al. Fluid resuscitation in severe sepsis and septic shock: systematic description of fluids used in randomized trials. Pol Arch Med Wewn 2013;123:603-8.

3. Rochwerg BZM, Millen T, Kavsak P, et al. Chloride concentration of IV albumin solutions available at Canadianhospitals. Canadian Critical Care Forum Abstract, 2015.

4. Yunos NM, Bellomo R, Hegarty C, et al. Association between a chloride-liberal vs chloride-restrictive intravenous fluid administration strategy and kidney injury in critically ill adults. JAMA 2012;308:1566-72.

5. Rochwerg B, Alhazzani W, Sindi A, et al. Fluid resuscitation in sepsis: a systematic review and network meta-analysis. Ann Intern Med 2014;161:347-55.

6. Young P, Bailey M, Beasley R, et al. Effect of a buffered crystalloid solution vs saline on acute kidney injury among patients in the intensive care unit: the SPLIT randomized clinical trial. JAMA 2015;314:1701-10.

7. McIntyre LA, Fergusson DA, Cook DJ, et al. Fluid resuscitation with $5 \%$ albumin versus normal saline in early septic shock: a pilot randomized, controlled trial. J Crit Care 2012;27:e311-6. 317.

8. Smith OM, McDonald E, Zytaruk N, et al. Enhancing the informed consent process for critical care research: strategies from a thromboprophylaxis trial. Intensive Crit Care Nurs 2013;29:300-9.

9. Kho ME, Molloy AJ, Clarke F, et al. CYCLE pilot: a protocol for a pilot randomised study of early cycle ergometry versus routine physiotherapy in mechanically ventilated patients. BMJ Open 2016;6:e011659.

10. Lamontagne F, Meade MO, Hébert PC, et al. Higher versus lower blood pressure targets for vasopressor therapy in shock: a multicentre pilot randomized controlled trial. Intensive Care Med 2016;42:542-50.

11. Lin CY, Chen YC. Acute kidney injury classification: AKIN and RIFLE criteria in critical patients. World J Crit Care Med 2012;1:40-5

12. Finfer $\mathrm{S}$. Reappraising the role of albumin for resuscitation. Curr Opin Crit Care 2013;19:315-20.

13. Morgan TJ. The ideal crystalloid - what is 'balanced'? Curr Opin Crit Care 2013;19:299-307.

14. Myburgh J. Advances in fluid resuscitation in critically ill patients: implications for clinical practice. Curr Opin Crit Care 2013;19:279-81.

15. Raghunathan K, Shaw AD, Bagshaw SM. Fluids are drugs: type, dose and toxicity. Curr Opin Crit Care 2013;19:290-8.

16. Seymour CW, Angus DC. Making a pragmatic choice for fluid resuscitation in critically ill patients. JAMA 2013;310:1803. 